\title{
GLOBAL UNIQUENESS FOR A TWO-DIMENSIONAL SEMILINEAR ELLIPTIC INVERSE PROBLEM
}

\author{
VICTOR ISAKOV AND ADRIAN I. NACHMAN
}

\begin{abstract}
For a general class of nonlinear Schrödinger equations $-\Delta u+a(x, u)$ $=0$ in a bounded planar domain $\Omega$ we show that the function $a(x, u)$ can be recovered from knowledge of the corresponding Dirichlet-to-Neumann map on the boundary $\partial \Omega$.
\end{abstract}

\section{INTRODUCTION}

Let $\Omega$ be a bounded Lipschitz domain in $\mathbb{R}^{2}$. For any real-valued potential $q(x)$ in $L^{p}(\Omega), p>1$, we denote by $\lambda_{1}(q)$ the lowest Dirichlet eigenvalue of $-\Delta+q$ in $\Omega$.

We consider the semilinear elliptic equation

$$
-\Delta u+a(x, u)=0 \quad \text { in } \Omega \text {. }
$$

We assume that, for some $p>1$,

$$
\begin{gathered}
a \in L^{p}\left(\Omega, C^{1}[-M, M]\right) \quad \text { for all } M<\infty, \\
a(x, 0) \equiv 0,
\end{gathered}
$$

and

$$
\frac{\partial a}{\partial u}(x, u) \geqq q_{*}(x) \text { for some } q_{*} \in L^{p}(\Omega) \text { with } \lambda_{1}\left(q_{*}\right)>0 .
$$

In view of (1.3), we can restate (1.2) more explicitly as follows: $\frac{\partial a}{\partial u}(x, u)$ is a Caratheodory function on $\Omega \times \mathbb{R}$ (i.e., measurable in $x$ and continuous in $u$ ) satisfying

$$
\sup _{|u| \leqq M}\left|\frac{\partial a}{\partial u}(\cdot, u)\right| \in L^{p}(\Omega) \quad \text { for all } M<\infty .
$$

One can then show (we do it in Section 2) that for any $f$ in $H^{1 / 2}(\partial \Omega) \cap C(\partial \Omega)$ there is a unique solution $u(x ; f)$ in $H^{1}(\Omega) \cap C(\bar{\Omega})$ of $(0.1)$ with $\left.u\right|_{\partial \Omega}=f$. We can therefore define the Dirichlet-to-Neumann map $\Lambda_{a}$ on the boundary $\partial \Omega$ by:

$$
\Lambda_{a} f=\left.\frac{\partial u}{\partial \nu}(\cdot ; f)\right|_{\partial \Omega} \in H^{-1 / 2}(\partial \Omega),
$$

Received by the editors August 22, 1994.

1991 Mathematics Subject Classification. Primary 35E30; Secondary 35J25, 35J10.

The first author was supported in part by NSF grant DMS-9101421.

The second author was supported in part by ONR grant N00014-91-1107. 
that is

$$
\left\langle v, \Lambda_{a} f\right\rangle_{\partial \Omega}=\int_{\Omega}[\nabla v(x) \cdot \nabla u(x)+v(x) a(x, u(x))] d x,
$$

where $u=u(\cdot ; f)$ and $v$ is any function in $H^{1}(\Omega)$.

We are interested in the inverse problem of determining the function $a(x, u)$ from knowledge of $\Lambda_{a}$.

In dimensions higher than 2, global uniqueness was proved in [Is-Sy] using a linearization technique first introduced in the context of parabolic equations in [Is 1]. (By the same technique, global uniqueness for $a(x, u)$ in the quasilinear equation $\operatorname{div}(a \operatorname{grad} u)=0$ was recently obtained in [S 2].)

In dimension 2 , the problem appears at first sight to be underdetermined: we wish to recover the function of three variables $a(x, u)$ from data on the two-dimensional set $\partial \Omega \times \partial \Omega$. It turns out that the nonlinear map $\Lambda_{a}$ contains knowledge on a full one-parameter family of Dirichlet-to-Neumann maps corresponding to linear Schrödinger operators. Global uniqueness for the formally determined linear case $a(x, u)=q(x) u$ has been unknown for a long time, although a number of partial results have been obtained: local uniqueness for potentials with small $H^{2}(\Omega)$ norm was proved by Sylvester and Uhlmann ([Sy-U]) and extended by Sun ([S1]) to potentials close to $q$ constant. Sun and Uhlmann ([Su-U1]) have proved local uniqueness near generic $q$ and global uniqueness for generic pairs of potentials; they have also shown ([Su-U2]) that $L^{\infty}$ potentials can be determined modulo $C^{\alpha}(\bar{\Omega}), 0 \leqq \alpha<1$. We will derive our results from the proof in [N 3] of global uniqueness of the conductivity coefficient $\gamma$ in the equation $\nabla \cdot(\gamma \nabla u)=0$ with a given Dirichlet-to-Neumann map.

To formulate our main result, the following notation will be helpful: let

$$
\begin{aligned}
& u_{*}(x)=\inf \left\{u(x ; f): f \in C(\partial \Omega) \cap H^{1 / 2}(\partial \Omega)\right\}, \\
& u^{*}(x)=\sup \left\{u(x ; f): f \in C(\partial \Omega) \cap H^{1 / 2}(\partial \Omega)\right\}
\end{aligned}
$$

(see also (4.12) and (4.17)) and let

$$
E=\left\{(x, u) \in \Omega \times \mathbb{R}: u_{*}(x)<u<u^{*}(x)\right\} .
$$

Theorem 1.1. Let $\Omega$ be a bounded Lipschitz domain in $\mathbb{R}^{2}$, and suppose that $a^{(1)}(x, u)$ and $a^{(2)}(x, u)$ satisfy the conditions (1.2), (1.3), and (1.4). If $\Lambda_{a^{(1)}}=$ $\Lambda_{a^{(2)}}$ then $u_{*}^{(1)}=u_{*}^{(2)}, u^{*(1)}=u^{*(2)}$ in $\Omega$ and $a^{(1)}=a^{(2)}$ on $E^{(1)}=E^{(2)}$.

The proof gives (at least when the boundary $\partial \Omega$ is $C^{1,1}$ ) a (theoretical) constructive procedure for recovering $u_{*}, u^{*}, E$ and the function $a(x, u)$ on $E$ from the Dirichlet-to-Neumann map $\Lambda_{a}$.

In the paper [Is-Sy] it was observed that in general, the set $E$ need not be all of $\Omega \times \mathbb{R}$. On the other hand, if we make the additional assumption

$$
\sup _{u \in \mathbb{R}} \frac{\partial a}{\partial u}(\cdot, u) \in L^{p}(\Omega) .
$$

then, as in [Is-Sy] we prove (for the larger class of nonlinear terms allowed here) that $E=\Omega \times \mathbb{R}$. 
Corollary 1.2. Let $\Omega$ be a bounded Lipschitz domain in $\mathbb{R}^{2}$. Suppose that the nonlinear coefficient $a$ in (1.1) satisfies the conditions (1.2), (1.3), (1.4) and (1.11). Then $a(x, u)$ can be recovered throughout $\Omega \times \mathbb{R}$ from knowledge of the Dirichlet-to-Neumann map $\Lambda_{a}$.

The above corollary is certainly applicable to linear equations, but in that case we have the following stronger uniqueness result, where only one of the two potentials is assumed a priori to satisfy (1.4). If $a(x, u)=q(x) u$ with $q$ such that 0 is not a Dirichlet eigenvalue of $-\Delta+q$ in $\Omega$, we denote by $\Lambda_{q}$ the corresponding (linear) Dirichlet-to-Neumann map.

Theorem 1.3. Let $\Omega$ be a bounded Lipschitz domain in $\mathbb{R}^{2}$ and let $q_{1}$ and $q_{2}$ be real-valued and in $L^{p}(\Omega)$ for some $p>1$. Suppose that $\lambda_{1}\left(q_{1}\right)>0$ and that 0 is not in the Dirichlet spectrum of $-\Delta+q_{2}$. If $\Lambda_{q_{1}}=\Lambda_{q_{2}}$, then $q_{1}=q_{2}$ a.e. in $\Omega$. If $\Omega$ is $C^{1,1}$ (or, more generally, if we know a $C^{1,1}$ domain $\Omega^{*} \supset \bar{\Omega}$ such that $q$, extended to be zero outside $\Omega$, has $\lambda_{1}(q)>0$ in $\left.\Omega^{*}\right)$ then the proof gives a constructive procedure to recover $q$ from knowledge of $\Lambda_{q}$ on $\partial \Omega$.

Theorem 1.3 yields the following semiglobal uniqueness results for fixedenergy inverse scattering problems. In the first of these, we consider pointsource data, measured in the "near-field": for a $q$ a real-valued potential (not necessarily of compact support) satisfying

$$
|q(x)| \leqq c(1+|x|)^{-1-\varepsilon} \text { for some } \varepsilon>0,
$$

let $\mathscr{G}_{q}(x, y ; \lambda)$ denote the outgoing solution in $\mathbb{R}^{2}$ of

$$
-\Delta_{x} \mathscr{G}_{q}(x, y ; \lambda)+(q(x)-\lambda) \mathscr{G}_{q}(x, y ; \lambda)=\delta(x-y) .
$$

Corollary 1.4. Let $\Omega$ be a bounded Lipschitz domain with connected exterior. Let $q_{1}, q_{2}$ be two real-valued potentials which satisfy (1.12) and are identical outside $\Omega$. If $\mathscr{G}_{q_{1}}(x, y ; \lambda)=\mathscr{G}_{q_{2}}(x, y ; \lambda)$ on $\partial \Omega \times \partial \Omega$ for one $\lambda>0$ with $\lambda<\lambda_{1}\left(q_{1}\right)$ in $\Omega$, then $q_{1}=q_{2}$.

Let $\mathscr{S}_{q}(\lambda)$ denote the single-layer potential operator defined on $\partial \Omega$ by

$$
\mathscr{S}_{q}(\lambda) f(x)=\int_{\partial \Omega} \mathscr{G}_{q}(x, y ; \lambda) f(y) d \sigma(y) .
$$

Under the hypotheses above, we show in the Appendix that $\mathscr{S}_{q_{j}}(\lambda)$ are bounded invertible operators: $H^{-1 / 2}(\partial \Omega) \rightarrow H^{1 / 2}(\partial \Omega)$ and the identity

$$
\Lambda_{q_{1}-\lambda}-\Lambda_{q_{2}-\lambda}=\mathscr{S}_{q_{1}}^{-1}(\lambda)-\mathscr{S}_{q_{2}}^{-1}(\lambda)
$$

(first found in [N1] for the case of constant background) holds. Furthermore, if $q$ is known outside $\Omega$ then the proof gives a formula (A.3) for recovery of $\Lambda_{q-\lambda}$, hence of $q$, from knowledge of $\mathscr{S}_{q}(\lambda)$ on $\partial \Omega$.

It may be worth noting that one motivation for working with the assumption $\lambda_{1}(q)>0$ throughout the paper, rather than the simpler $q(x) \geqq 0$, is to allow Corollary 1.4 to be applicable to the acoustic equation. Let $\mathscr{G}(x, y)$ denote the wave-field generated by a point source oscillating harmonically with frequency $\omega$ in a medium with variable speed $c(x)$ :

$$
\Delta_{x} \mathscr{G}(x, y)+\frac{\omega^{2}}{c^{2}(x)} \mathscr{G}(x, y)=-\delta(x-y) .
$$


Assuming for simplicity $c(x) \equiv c_{0}$ (a known constant) outside $\Omega$, we have $\lambda=\frac{\omega^{2}}{c_{0}^{2}}$ and $q(x)=\omega^{2}\left(\frac{1}{c_{0}^{2}}-\frac{1}{c^{2}(x)}\right)$, so that $q(x)-\lambda$ cannot be positive in this case. The condition $\lambda_{1}(q)>\lambda$ becomes $\lambda_{1}\left(-\omega^{2} / c^{2}(x)\right)>0$. A sufficient condition for the latter is $c^{2}(x) \geqq \omega^{2} /\left(\lambda_{1}^{0}-\varepsilon\right)$ for some $\varepsilon>0$, with $\lambda_{1}^{0}$ the first Dirichlet eigenvalue for the Laplacian in $\Omega$.

Corollary 1.4 is in turn equivalent to the following far-field version.

Corollary 1.5. Let $\Omega$ be a bounded Lipschitz domain with $\mathbb{R}^{2} \backslash \bar{\Omega}$ connected. Let $q_{1}, q_{2}$ be two real-valued potentials which satisfy (1.12) and are identical outside $\Omega$. Assume $\lambda_{1}\left(q_{1}\right)>\lambda>0$. If the corresponding scattering amplitudes at the energy $\lambda$ satisfy $A_{1}\left(\theta^{\prime}, \theta ; \lambda\right)=A_{2}\left(\theta^{\prime}, \theta ; \lambda\right)$ for all incident and outgoing directions $\theta, \theta^{\prime}$, then $q_{1} \equiv q_{2}$.

The theoretical equivalence of the data in Corollaries 1.4 and 1.5 (via explicit formulae) goes back to [B], at least when $\Omega$ is a disk and $q$ vanishes outside $\Omega$. In the Appendix (Proposition A.2) we give a proof for the more general case above, based on an identity first introduced in [N2].

Uniqueness in the two-dimensional inverse scattering problem at fixed energy, for exponentially decaying potentials assumed sufficiently small, was obtained by Novikov ([No]). More recently, in [Is Su] a global uniqueness result was proved assuming the scattering amplitude given at a finite (sufficiently large) number of energies.

The plan of the remainder of this paper is the following: in Section 2 we prove the unique solvability of the Dirichlet problem for the nonlinear equation (1.1); in Section 3 we treat the linear case, Theorem 1.3. In Section 4 we prove Theorem 1.1, Corollary 1.2 and conclude with a summary of the main steps of our reconstruction procedure. The Appendix is devoted to the inverse scattering results, Corollaries 1.4 and 1.5.

\section{The Dirichlet PROBLEM}

In this section we prove the unique solvability of the Dirichlet problem for the equation (1.1) in a Lipschitz domain, and a comparison principle.

Proposition 2.1. Let $\Omega$ be a bounded Lipschitz domain in $\mathbb{R}^{2}$. If $a(x, u)$ satisfies the conditions (1.2) and (1.4), then for any $f \in H^{1 / 2}(\partial \Omega) \cap C(\partial \Omega)$ there is a unique $u(\cdot ; f) \in H^{1}(\Omega) \cap C(\bar{\Omega})$ solution of $(1.1)$ with $\left.u\right|_{\partial \Omega}=f$. Furthermore, we have the bound

$$
\sup _{\Omega}|u(\cdot ; f)| \leqq C\|f\|_{L^{\infty}(\partial \Omega)}
$$

with $C$ depending only on $q_{*}$ and $\Omega$.

Proof. 1. We begin with a substitution which will change the nonlinear term in (1.1) to one which is nondecreasing in $u$, thereby allowing the use of the maximum principle. Define $q_{*}(x) \equiv 0$ outside $\Omega$; let $\tilde{\Omega}$ be a smooth bounded domain containing $\bar{\Omega}$ and so close to it that the corresponding first eigenvalue $\tilde{\lambda}_{1}\left(q_{*}\right)$ satisfies $\tilde{\lambda}_{1}\left(q_{*}\right)>\frac{1}{2} \lambda_{1}\left(q_{*}\right)>0$. The Dirichlet problem

$$
-\Delta u^{+}+q_{*} u^{+}=0 \text { in } \tilde{\Omega}, \quad u^{+}=1 \text { on } \partial \tilde{\Omega}
$$

then has a positive solution $u^{+}$, continuous in $\overline{\tilde{\Omega}}$ (see for instance [A-S], appendix); since $\Delta u^{+} \in L^{p}(\tilde{\Omega})$ and $\partial \tilde{\Omega}$ is smooth we also have $u^{+} \in W^{2, p}(\tilde{\Omega})$. 
To solve (1.1) in $\Omega$, we substitute $u=u^{+} v$. The equation for $v$ then becomes

$$
L v+b(x, v)=0 \text { in } \Omega, \quad v=g \text { on } \partial \Omega,
$$

with $g=f / u^{+}$,

$$
L v=-\Delta v-2 \frac{\nabla u^{+}}{u^{+}} \cdot \nabla v
$$

and

$$
b(x, v)=\frac{1}{u^{+}(x)} a\left(x, u^{+}(x) v\right)-q_{*}(x) v .
$$

Note that the new nonlinear term satisfies $\frac{\partial b}{\partial v} \geqq 0$. Also, $\sup _{|v| \leqq M}|b(\cdot, v)|$ is in $L^{p}(\Omega)$, while the coefficient $\nabla u^{+} / u^{+}$of $L$ is in $L^{\tilde{p}}(\Omega), \tilde{p}>2$, by Sobolev imbedding.

2. For any $F \in L^{p}(\Omega)$ there is a unique $w \in C(\bar{\Omega}) \cap H^{1}(\Omega)$ solution of the linear Dirichlet problem $L w=F$ in $\Omega,\left.w\right|_{\partial \Omega}=g$. We henceforth fix $g$, write $w=L^{-1} F$ and claim that $L^{-1}$ is compact as a map from $L^{p}(\Omega)$ to $C(\bar{\Omega})$. To see this, first extend $F$ to be zero in $\tilde{\Omega} \backslash \Omega$ and let $w_{0}$ be the solution of $L w_{0}=F$ in $\tilde{\Omega}$ with $\left.w_{0}\right|_{\partial \tilde{\Omega}}=0$. Then

$$
-\Delta\left(w_{0} u^{+}\right)+q_{*}\left(w_{0} u^{+}\right)=F u^{+},
$$

and it follows that $w_{0} \in W^{2, p}(\tilde{\Omega})$; hence the map $F \rightarrow w_{0}$ from $L^{p}(\Omega)$ to $C(\tilde{\Omega})$ is compact. Next, let $w_{1} \in H^{1}(\Omega) \cap C(\bar{\Omega})$ be the solution of $L w_{1}=0$ in $\Omega$ with $w_{1}=g-w_{0}$ on $\partial \Omega$. If $\left\{F^{(k)}\right\}$ is a bounded sequence in $L^{p}(\Omega)$, then, since the operator $F \rightarrow w_{0}$ is compact, we can find a subsequence $k_{n}$ so that $\left\{w_{0}^{k_{n}}\right\}$ converges in $C(\tilde{\Omega})$. By the weak maximum principle for $L$ the same will be true for the corresponding $\left\{w_{1}^{\left(k_{n}\right)}\right\}$, thus proving the compactness of $L^{-1}$.

3. We now return to the nonlinear equation (2.3). Let $M=\sup _{\partial \Omega}|g|$ and consider the modified coefficient $b_{M}$ given by

$$
b_{M}(x, v)=b\left(x, \psi_{M}(v)\right)
$$

with

$$
\psi_{M}(v)=v \text { if }|v| \leqq 2 M, \psi_{M}(v)=2 \operatorname{sgn} v M \text { if }|v|>2 M .
$$

Let $T$ be the operator on $C(\bar{\Omega})$ defined as $T v=L^{-1}\left(-b_{M}(x, v(x))\right)$ and let

$$
\rho=\left\|\sup _{|v| \leqq M}|b(\cdot, v)|\right\|_{L^{p}(\Omega)}\left\|L^{-1}\right\|_{L^{p}(\Omega) \rightarrow C(\Omega)} .
$$

Then $T$ is compact and maps the ball $\left\{v \in C(\bar{\Omega}): \sup _{\bar{\Omega}}|v| \leqq \rho\right\}$ to itself. We may conclude from the Schauder fixed point theorem that $T$ has a fixed point $v$. By construction, we have $v \in C(\Omega) \cap H^{1}(\Omega)$,

$$
L v+b_{M}(x, v)=0 \text { and }\left.v\right|_{\partial \Omega}=g .
$$

The maximum principle now shows that $\sup _{\bar{\Omega}}|v| \leqq M$. Therefore $b_{M}(x, v)=$ $b(x, v)$ in $\Omega$, so that $v$ solves (2.2), as required. The corresponding solution $u$ of (1.1) satisfies

$$
\sup _{\Omega}|u| \leqq\left[\sup _{\Omega} u^{+} / \inf _{\partial \Omega} u^{+}\right]\|f\|_{L^{\infty}(\partial \Omega)}
$$


which clearly holds uniformly for all $a(x, u)$ satisfying $\frac{\partial a}{\partial u} \geqq q_{*}$.

4. It remains to verify uniqueness. Let $u_{1}, u_{2}$ in $H^{1}(\Omega) \cap C(\bar{\Omega})$ be two solutions of (1.1) with $\left.u_{1}\right|_{\partial \Omega}=\left.u_{2}\right|_{\partial \Omega}$. Then, writing

$$
a\left(x, u_{1}(x)\right)-a\left(x, u_{2}(x)\right)=q_{12}(x)\left(u_{1}(x)-u_{2}(x)\right)
$$

with

$$
q_{12}(x)=\int_{0}^{1} \frac{\partial a}{\partial u}\left(x, u_{2}(x)+t\left(u_{1}(x)-u_{2}(x)\right)\right) d t,
$$

we have $q_{12} \geqq q_{*}$ so that $\lambda_{1}\left(q_{12}\right)>0$. The function $w(x)=u_{1}(x)-u_{2}(x)$ satisfies $-\Delta w+q_{12} w=0$ in $\Omega$ and $w=0$ on $\partial \Omega$; thus $w \equiv 0$ since 0 is below the Dirichlet spectrum of $q_{12}$.

We conclude this section with the observation (which will be helpful in Section 4) that our assumptions (1.2) and (1.4) on $a(x, u)$ also suffice for the following comparison principle (usually stated for $a$ increasing in $u$ ).

Lemma 2.2. Under the hypotheses of Proposition 2.1, if $f_{j} \in H^{1 / 2}(\partial \Omega) \cap C(\partial \Omega)$ satisfy $f_{1} \leqq f_{2}$ on $\partial \Omega$ then for the corresponding solutions $u\left(\cdot ; f_{j}\right)$ we have

$$
u\left(x, f_{1}\right) \leqq u\left(x, f_{2}\right) \quad \text { in } \Omega .
$$

Proof. With $u^{+}$as in the proof of Proposition 2.1, let $v_{j}(x)=u\left(x ; f_{j}\right) / u^{+}(x)$. Then the functions $v_{j}$ satisfy equation (2.3), hence for $v_{1}-v_{2}$ we have

$$
L\left(v_{1}-v_{2}\right)+c(x)\left(v_{1}-v_{2}\right)=0 \quad \text { in } \Omega,
$$

with

$$
c(x)=\int_{0}^{1} \frac{\partial b}{\partial v}\left(x, t v_{1}(x)+(1-t) v_{2}(x)\right) d t \geqq 0 .
$$

The weak maximum principle for the linear equation (2.15) then implies $v_{1}-$ $v_{2} \leqq 0$ in $\Omega$, hence also $(2.14)$, since $u^{+}$is positive throughout $\bar{\Omega}$.

\section{THE LINEAR CASE}

Throughout this section we assume $a(x, u)=q(x) u$ with $q \in L^{p}(\Omega), p>1$. Theorem 1.3 will be obtained as a consequence of a number of facts proved in [N3]. We briefly recall the relevant notation. $\partial \Omega$ :

For any $k \in \mathbb{C} \backslash 0$ we denote by $S_{k}$ the following single-layer operator on

$$
S_{k} f(x)=\int_{\partial \Omega} G_{k}(x-y) f(y) d \sigma(y),
$$

with $G_{k}$ the zero-energy Faddeev Green's function

$$
G_{k}(x)=\frac{e^{i\left(x_{1}+i x_{2}\right) k}}{(2 \pi)^{2}} \int \frac{e^{i x \cdot \xi}}{|\xi|^{2}+2 k\left(\xi_{1}+i \xi_{2}\right)} d \xi .
$$

A family $\psi_{q}(x, k)$ of solutions of the Schrödinger equation in all of $\mathbb{R}^{2}$

$$
(-\Delta+q) \psi_{q}(x, k)=0,
$$


is constructed by solving (when possible) the Fredholm integral equations

$$
\psi_{q}(x, k)=e^{i\left(x_{1}+i x_{2}\right) k}-G_{k} *\left(q \psi_{q}(\cdot, k)\right) .
$$

If $k \in \mathbb{C} \backslash 0$ is such that (3.3) is not uniquely solvable (in an appropriate weighted Sobolev space) then it is called an exceptional point. For non-exceptional $k$ we define the scattering transform $t_{q}$ of $q$ by

$$
t_{q}(k)=\int_{\mathbb{R}^{2}} e^{i\left(x_{1}-i x_{2}\right) \bar{k}} q(x) \psi_{q}(x, k) d x .
$$

We prove below that if $\lambda_{1}\left(q_{1}\right)>0$ then $q_{1}$ can be extended to a potential in $L^{p}\left(\mathbb{R}^{2}\right)$, of compact support, which has no (zero-energy) exceptional points. Moreover, the same will be true of $q_{2}$ if $\Lambda_{q_{2}}=\Lambda_{q_{1}}$. We can then use the method of [N3] to, on the one hand, obtain $t_{q_{1}}(k)=t_{q_{2}}(k)$ for all $k \in \mathbb{C} \backslash 0$ from knowledge of the Dirichlet-to-Neumann map and, on the other, to recover $q_{1}=q_{2}$ from their common transform $t$.

We begin with a simple lemma which will allow us to work in a slightly larger domain $\tilde{\Omega}$.

Lemma 3.1. Let $\Omega, \tilde{\Omega}$ be bounded Lipschitz domains with $\bar{\Omega} \subset \bar{\Omega}$, and let $q_{1}, q_{2} \in L^{p}(\tilde{\Omega})$. Assume that zero is not a Dirichlet eigenvalue of $-\Delta+q_{1}$ in $\Omega$, $\tilde{\Omega}$ or of $-\Delta+q_{2}$ in $\Omega$. If $q_{1} \equiv q_{2}$ in $\tilde{\Omega} \backslash \bar{\Omega}$ and $\Lambda_{q_{1}}=\Lambda_{q_{2}}$ on $H^{1 / 2}(\partial \Omega) \cap C(\partial \Omega)$ then zero is not a Dirichlet eigenvalue of $-\Delta+q_{2}$ in $\tilde{\Omega}$ and (for the corresponding Dirichlet-to-Neumann maps on $\partial \tilde{\Omega}$ ) we have $\tilde{\Lambda}_{q_{1}}=\tilde{\Lambda}_{q_{2}}$ on $H^{1 / 2}(\partial \tilde{\Omega})$.

Proof. 1. If we had a $v_{2}$ such that $\left(-\Delta+q_{2}\right) v_{2}=0$ in $\tilde{\Omega},\left.v_{2}\right|_{\partial \tilde{\Omega}}=0$, we could define $v_{1}$ in $\tilde{\Omega}$ to equal $v_{2}$ in $\tilde{\Omega} \backslash \bar{\Omega}$ and equal to the solution of the Dirichlet problem $\left(-\Delta+q_{1}\right) v_{1}=0,\left.v_{1}\right|_{\partial \Omega}=\left.v_{2}\right|_{\partial \Omega}$ in $\Omega$. Then (since $q_{1} \equiv q_{2}$ outside $\Omega$ and $\Lambda_{q_{1}}=\Lambda_{q_{2}}$ on $\left.\partial \Omega\right) v_{1}$ would be a solution of $\left(-\Delta+q_{1}\right) v_{1}=0$ throughout $\tilde{\Omega}$ with $\left.v_{1}\right|_{\partial \tilde{\Omega}}=0$, contradicting our hypothesis on $q_{1}$. Thus 0 is not a Dirichlet eigenvalue of $q_{2}$ in $\Omega$.

2. For any $f_{1}, f_{2} \in H^{1 / 2}(\partial \tilde{\Omega})$ we now let $u_{j}$ be the unique $H^{1}(\tilde{\Omega})$ solutions of the Dirichlet problems $\left(-\Delta+q_{j}\right) u_{j}=0$ in $\tilde{\Omega}$ with $u_{j}=f_{j}$ on $\partial \tilde{\Omega}, j=1,2$. From (1.7) and the symmetry of $\tilde{\Lambda}_{q_{2}}$ we have Alessandrini's identity

$$
\left\langle f_{2},\left(\tilde{\Lambda}_{q_{2}}-\tilde{\Lambda}_{q_{1}}\right) f_{1}\right\rangle_{\partial \tilde{\Omega}}=\int_{\tilde{\Omega}}\left(q_{2}-q_{1}\right) u_{1} u_{2}
$$

Since, by assumption, $q_{1}-q_{2} \equiv 0$ outside $\Omega$, (3.6) yields

$$
\left\langle f_{2},\left(\tilde{\Lambda}_{q_{2}}-\tilde{\Lambda}_{q_{1}}\right) f_{1}\right\rangle_{\partial \tilde{\Omega}}=\int_{\Omega}\left(q_{2}-q_{1}\right) u_{1} u_{2}=\left\langle u_{2},\left(\Lambda_{q_{2}}-\Lambda_{q_{1}}\right) u_{1}\right\rangle_{\partial \Omega}
$$

(the latter by the above identity in $\Omega$ ). Note that $u_{1} \in W_{\text {loc }}^{2, p}$ in the interior of $\tilde{\Omega}$; in particular, $u_{1}$ is continuous on $\partial \Omega$. Thus the right side of (3.7) is zero, by hypothesis, and $\tilde{\Lambda}_{q_{2}}=\tilde{\Lambda}_{q_{1}}$, as claimed.

Remark. The proof of Proposition 6.1 in [N3], with the obvious modifications to the case of Schrödinger operators considered here, gives a constructive way to determine $\tilde{\Lambda}_{q}$ on $H^{1 / 2}(\partial \tilde{\Omega})$ from knowledge of $\Lambda_{q}$ on $H^{1 / 2}(\partial \Omega) \cap C(\partial \Omega)$ if $q$ is known in $\tilde{\Omega} \backslash \bar{\Omega}$. 
Proof of Theorem 1.3. We make a preliminary extension of $q_{1}$ and $q_{2}$ by defining them to be identically zero outside $\Omega$. As in Section 2 , we then let $\Omega^{*}$ be a smooth bounded domain containing $\bar{\Omega}$ and sufficiently close to it so that $\lambda_{1}^{*}\left(q_{1}\right)>0$. Next, let $\tilde{\Omega}$ be any bounded smooth (or, more generally, $C^{1,1}$ ) domain containing $\bar{\Omega}^{*}$. The following elementary lemma will enable us to appropriately define the extension of $q_{1}$ and $q_{2}$ in $\tilde{\Omega} \backslash \Omega^{*}$.

Lemma 3.2. Let $\Omega^{*}, \tilde{\Omega}$ be bounded $C^{1,1}$ domains with $\overline{\Omega^{*}} \subset \tilde{\Omega}$ and let $q_{1} \in$ $L^{p}\left(\Omega^{*}\right)$. Given $\Lambda_{q_{1}}^{*}$ on $\partial \Omega^{*}$, we can construct a function $\psi_{0} \in H^{2}\left(\tilde{\Omega} \backslash \bar{\Omega}^{*}\right)$ which is bounded away from zero, identically equal to one near $\partial \tilde{\Omega}$ and such that

$$
\left.\psi_{0}\right|_{\partial \Omega^{*}}=1,\left.\quad \frac{\partial \psi_{0}}{\partial \nu}\right|_{\partial \Omega^{*}}=\Lambda_{q_{1}}^{*} 1
$$

Proof of Lemma 3.2. By elliptic regularity, $\Lambda_{q_{1}}^{*} 1 \in H^{1 / 2}\left(\partial \Omega^{*}\right)$. We first choose $\psi_{*} \in H^{2}\left(\tilde{\Omega} \backslash \bar{\Omega}^{*}\right)$ with $\left.\psi_{*}\right|_{\partial \Omega^{*}}=1,\left.\frac{\partial \psi_{*}}{\partial \nu}\right|_{\partial \Omega^{*}}=\Lambda_{q_{1}}^{*} 1$. Since $\psi_{*}$ is continuous, there are neighborhoods $U_{0}, U_{1}$ of $\partial \Omega^{*}$ with $\bar{U}_{0} \subset U_{1} \subset \bar{U}_{1} \subset \tilde{\Omega}$ such that $\psi_{*}>\frac{3}{4}$ in $U_{0}, \psi_{*}>\frac{1}{2}$ in $U_{1}$. Let $\chi$ be smooth, of compact support in $U_{1}, 0 \leqq \chi \leqq 1$, with $\chi \equiv 1$ in $\bar{U}_{0}$. Define $\psi_{0}=(1-\chi)+\chi \psi_{*}$. Then $\psi_{0} \equiv \psi_{*}$ in $\bar{U}_{0}$ so that (3.8) are valid for $\psi_{0}$. Also, $\psi_{0} \equiv 1$ outside $U_{1}$ and $\psi_{0}>\frac{1}{3}$ throughout $\tilde{\Omega} \backslash \Omega^{*}$.

Proof of Theorem 1.3 (continued). With $\psi_{0}$ as in the above lemma, we extend $q_{1}$ and $q_{2}$ to all of $\mathbb{R}^{2}$ by

$$
q_{1}=q_{2}=\frac{\Delta \psi_{0}}{\psi_{0}} \text { in } \tilde{\Omega} \backslash \bar{\Omega}^{*} \text { and } q_{1}=q_{2} \equiv 0 \text { in }\left(\mathbb{R}^{2} \backslash \tilde{\Omega}\right) \cup\left(\bar{\Omega}^{*} \backslash \Omega\right) \text {. }
$$

If we define $\psi_{0}$ inside $\Omega^{*}$ to be the solution of $\left(-\Delta+q_{1}\right) \psi_{0}=0$ with $\left.\psi_{0}\right|_{\partial \Omega^{*}}=$ 1 , and outside $\tilde{\Omega}$ to be identically 1 , then, in view of (3.8) and (3.9) we have $\left(-\Delta+q_{1}\right) \psi_{0}=0$ throughout $\mathbb{R}^{2}, \psi_{0}-1 \in W^{2, p}\left(\mathbb{R}^{2}\right)$. By the assumption $\lambda_{1}\left(q_{1}\right)>0$ in $\Omega^{*}$ and the construction in Lemma 3.2, $\psi_{0}$ is also bounded away from zero. Theorem 3 of [N3] now shows that $q_{1}$ has no (zero-energy) exceptional points and $t_{q_{1}}(k)=O\left(|k|^{\varepsilon}\right)$ for $k$ near zero. Thus, by Theorem 5 of [N3] the integral equation on $\partial \tilde{\Omega}$ :

$$
\psi_{q}(\cdot, k)=e^{i z k}-\tilde{S}_{k}\left(\tilde{\Lambda}_{q_{1}}-\tilde{\Lambda}_{0}\right) \psi_{q}(\cdot, k)
$$

is uniquely solvable for any $k \in \mathbb{C} \backslash 0$. Since we've defined $q_{2} \equiv q_{1}$ in $\tilde{\Omega} \backslash \Omega$, we have $\tilde{\Lambda}_{q_{1}}=\tilde{\Lambda}_{q_{2}}$, by Lemma 3.1. The converse of Theorem 5(iii) in [N3] now shows that $q_{2}$ is also free of exceptional points, so that its scattering transform $t_{q_{2}}(k)$ is well defined on $\mathbb{C} \backslash 0$. Furthermore, from $\tilde{\Lambda}_{q_{1}}=\tilde{\Lambda}_{q_{2}}$ and (3.10) we have $\psi_{q_{1}}(x, k)=\psi_{q_{2}}(x, k)$ for $x$ on $\partial \tilde{\Omega}$ and all $k \neq 0$, hence also $t_{q_{1}} \equiv t_{q_{2}}$ in view of the formula (see Theorem 5(iv) in [N3]):

$$
t_{q}(k)=\left\langle e^{i \bar{z} \bar{k}},\left(\tilde{\Lambda}_{q}-\tilde{\Lambda}_{0}\right) \psi_{q}(\cdot, k)\right\rangle_{\partial \tilde{\Omega}} .
$$

Theorem 4.1 of [N3] now shows that $\psi_{q_{1}}(x, k)=\psi_{q_{2}}(x, k)$ for all $(x, k)$ and that these functions never vanish. Returning to (3.3) we obtain $q_{1}=q_{2}$.

The above proof gives the following procedure for reconstructing $q$ from knowledge of $\Lambda_{q}$ on $\partial \Omega$. We assume $\Omega^{*}$ given (as in the statement of Theorem 
1.3). We first extend $q$ to be zero in $\Omega^{*} \backslash \Omega$ and determine $\Lambda_{q}^{*} 1$. (See the Remark after the proof of Lemma 3.1.) If $\partial \Omega$ is $C^{1,1}$ to begin with, this step is not needed. Next, we choose $\tilde{\Omega} \supset \bar{\Omega}^{*}$ and extend $q$ to be $\Delta \psi_{0} / \psi_{0}$ in $\tilde{\Omega} \backslash \Omega^{*}$, with $\psi_{0}$ constructed as in Lemma 3.2 ; this allows us to determine $\tilde{\Lambda}_{q}$ on $\partial \tilde{\Omega}$. With these preliminary adjustments completed, we can now solve equation (3.10) to find $\psi(\cdot, k)$ on $\partial \tilde{\Omega}$ and obtain $t_{q}(k)$ by formula (3.11). The Fredholm integral equation in the proof of Theorem 4.1 of [N3] then yields $\psi(x, k)$, hence $q$.

\section{LINEARIZATION}

To prove Theorem 1.1 we combine the result in the previous section for the linear case with the following.

Lemma 4.1. Suppose $\Omega$ is a bounded Lipschitz domain and $a(x, u)$ satisfies (1.2) and (1.4). Then for any $f_{0}, f \in H^{1 / 2}(\partial \Omega) \cap C(\partial \Omega)$ we have

$$
\lim _{\varepsilon \rightarrow 0} \frac{\Lambda_{a}\left(f_{0}+\varepsilon f\right)-\Lambda_{a}\left(f_{0}\right)}{\varepsilon}=\Lambda_{q\left(\cdot ; f_{0}\right)}(f)
$$

in the $H^{-1 / 2}(\partial \Omega)$ norm, where the potential $q\left(\cdot ; f_{0}\right)$ is defined to be

$$
q\left(x ; f_{0}\right)=\frac{\partial a}{\partial u}\left(x, u\left(x ; f_{0}\right)\right) .
$$

Proof. Fix $f_{0}, f$ and denote by $u_{\varepsilon}$ the function $u_{\varepsilon}(x)=u\left(x ; f_{0}+\varepsilon f\right)$. Since

$$
-\Delta\left(u_{\varepsilon}-u_{0}\right)+a\left(x, u_{\varepsilon}\right)-a\left(x, u_{0}\right)=0,
$$

the function $u_{\varepsilon}-u_{0}$ is a solution of the Dirichlet problem

$$
-\Delta\left(u_{\varepsilon}-u_{0}\right)+q_{\varepsilon}(x)\left(u_{\varepsilon}-u_{0}\right)=0 \text { in } \Omega, \quad u_{\varepsilon}-u_{0}=\varepsilon f \text { on } \partial \Omega,
$$

with

$$
q_{\varepsilon}(x)=: \int_{0}^{1} \frac{\partial a}{\partial u}\left(x, t u_{\varepsilon}(x)+(1-t) u_{0}(x)\right) d t .
$$

From the inequality $q_{\varepsilon}(x) \geqq q_{*}(x)$ and the estimate $(2.11)$ we obtain

$$
\sup _{\Omega}\left|u_{\varepsilon}-u_{0}\right| \leqq C \varepsilon\|f\|_{L^{\infty}(\partial \Omega)}
$$

with $C$ independent of $\varepsilon$. It then follows from the continuity of $\frac{\partial a}{\partial u}$ in $u$, (1.5), and dominated convergence that

$$
\lim _{\varepsilon \rightarrow 0}\left\|q_{\varepsilon}-q_{0}\right\|_{L^{p}(\Omega)}=0 .
$$

Now let $v_{\varepsilon}$ denote the difference quotient $v_{\varepsilon}=\frac{1}{\varepsilon}\left(u_{\varepsilon}-u_{0}\right)$ and let $v_{0}$ be the solution of

$$
-\Delta v_{0}+q_{0} v_{0}=0, \text { with }\left.v_{0}\right|_{\partial \Omega}=f .
$$

Then $v_{\varepsilon}-v_{0} \in H_{0}^{1}(\Omega)$ solves

$$
-\Delta\left(v_{\varepsilon}-v_{0}\right)+q_{0}\left(v_{\varepsilon}-v_{0}\right)=\left(q_{0}-q_{\varepsilon}\right) v_{\varepsilon} .
$$

The right side of (4.9) tends to zero in $L^{p}(\Omega)$, in view of (4.6) and (4.7); since $q_{0} \geqq q_{*}$, strict coercivity yields

$$
\lim _{\varepsilon \rightarrow 0}\left\|v_{\varepsilon}-v_{0}\right\|_{H^{1}(\Omega)}=0 .
$$


For any $w \in H^{1}(\Omega)$ we have

$$
\begin{aligned}
\mid\langle w, & \left.\frac{\Lambda_{a}\left(f_{0}+\varepsilon f\right)-\Lambda_{a}\left(f_{0}\right)}{\varepsilon}-\Lambda_{q_{0}}\left(f_{0}\right)\right\rangle \mid \\
& =\left|\int_{\Omega} \nabla w \cdot \nabla\left(v_{\varepsilon}-v_{0}\right)+w\left(q_{\varepsilon} v_{\varepsilon}-q_{0} v_{0}\right)\right| \\
& \leqq C\|w\|_{H^{1}(\Omega)}\left(\left\|v_{\varepsilon}-v_{0}\right\|_{H^{1}(\Omega)}+\left\|q_{\varepsilon}-q_{0}\right\|_{L^{p}(\Omega)}\right)
\end{aligned}
$$

with $C$ independent of $\varepsilon$, and (4.1) follows from (4.7) and (4.10).

In our inversion procedure it will suffice to work with functions $f_{0}(x) \equiv \theta$ constant on $\partial \Omega$. We also note, as in [Is-Sy], that Lemma 2.2 yields the following simpler formulae for the functions $u_{*}, u^{*}$ defined in (1.8), (1.9):

$$
u_{*}(x)=\inf _{\theta \in \mathbb{R}} u(x ; \theta), \quad u^{*}(x)=\sup _{\theta \in \mathbb{R}} u(x ; \theta),
$$

since for any $f \in H^{1 / 2}(\partial \Omega) \cap C(\partial \Omega)$ we have

$$
u\left(x ; \min _{\partial \Omega} f\right) \leqq u(x ; f) \leqq u\left(x ; \max _{\partial \Omega} f\right) .
$$

Proof of Theorem 1.1. The proof of Lemma 4.1 (with $f_{0} \equiv \theta$ and $f \equiv 1$ ) shows that $u(x ; \theta)$ is differentiable in $\theta$ (in the $H^{1}(\Omega)$ topology) and $\frac{\partial u}{\partial \theta}(x ; \theta)$ is the solution of the linear Dirichlet problem

$$
(-\Delta+q(x ; \theta)) \frac{\partial u}{\partial \theta}(x ; \theta)=0 \text { in } \Omega, \quad \frac{\partial u}{\partial \theta}(x ; \theta) \equiv 1 \text { on } \partial \Omega,
$$

with

$$
q(x ; \theta)=\frac{\partial a}{\partial u}(x, u(x ; \theta)) .
$$

Note that, since $u(\cdot ; \theta)$ is bounded, $q(\cdot ; \theta)$ is in $L^{p}(\Omega)$ for any $\theta$; also $\lambda_{1}(q(\cdot ; \theta))>0$, since $q(\cdot ; \theta) \geqq q_{*}$.

Given $\Lambda_{a}$ on $H^{1 / 2}(\partial \Omega) \cap C(\partial \Omega)$ we can, in view of Lemma 4.1, determine $\Lambda_{q(\cdot ; \theta)}$ on $H^{1 / 2}(\partial \Omega) \cap C(\partial \Omega)$ for any $\theta \in \mathbb{R}$. By the inversion method for the linear case (Section 3 ) we can then reconstruct the potentials $q(\cdot ; \theta)$. Solving (4.14) then yields $\frac{\partial u}{\partial \theta}(x ; \theta)$ on $\Omega \times \mathbb{R}$. We know (from (1.3) and uniqueness for $(1.1))$ that $u(x ; 0)=0$, thus we also obtain, for all $(x, \theta)$ in $\Omega \times \mathbb{R}$

$$
u(x ; \theta)=\int_{0}^{\theta} \frac{\partial u}{\partial \theta}(x ; s) d s .
$$

In particular, the functions $u^{*}(x)$ and $u_{*}(x)$ (hence also the set $E$ ) are recovered: noting that $\frac{\partial u}{\partial \theta}(x ; \theta)>0\left(\right.$ since $\left.\lambda_{1}(q(\cdot ; \theta))>0\right)$ we in fact have

$$
u^{*}(x)=\int_{0}^{\infty} \frac{\partial u}{\partial \theta}(x ; \theta) d \theta \text { and } u_{*}(x)=-\int_{-\infty}^{0} \frac{\partial u}{\partial \theta}(x ; \theta) d \theta
$$

For every $x$, the function $u(x ; \theta)$ is strictly increasing in $\theta$; this allows us to define for $u \in\left(u_{*}(x), u^{*}(x)\right)$ the inverse function $\theta(x, u)$. Then

$$
\frac{\partial a}{\partial u}(x, u)=q(x ; \theta(x, u))
$$


and (in view of (1.3)) we finally obtain $a(x, u)$ on $E$ as:

$$
a(x, u)=\int_{0}^{u} q(x ; \theta(x, s)) d s=\int_{0}^{\theta(x, u)} q(x ; \theta) \frac{\partial u}{\partial \theta}(x ; \theta) d \theta .
$$

Proof of Corollary 1.2. Let $q^{*}$ denote the potential

$$
q^{*}(x)=: \sup _{u \in \mathbb{R}} \frac{\partial a}{\partial u}(x, u)
$$

and let $u^{*}$ be the solution of $\left(-\Delta+q^{*}\right) u^{*}=0$ in $\Omega$ with $\left.u^{*}\right|_{\partial \Omega}=1$. Consider the function

$$
w(x, \theta)=\left(\frac{\partial u}{\partial \theta}(x ; \theta)-u^{*}(x)\right) / u^{+}(x),
$$

with $u^{+}$as in the proof of Proposition 2.1. We have $\left.w\right|_{\partial \Omega}=0$, and

(4.22) $-\Delta w-2 \frac{\nabla u^{+}}{u^{+}} \cdot \nabla w+\left(q(x ; \theta)-q_{*}(x)\right) w=\left(q^{*}(x)-q(x, \theta)\right) \frac{u^{*}(x)}{u^{+}(x)} \geqq 0$.

The weak maximum principle yields $w \geqq 0$ in $\Omega$, hence

$$
\frac{\partial u}{\partial \theta}(x ; \theta) \geqq \inf _{\Omega} u^{*}>0 \text { for all }(x, \theta) \in \Omega \times \mathbb{R} .
$$

From (4.17) it now follows that $u^{*}(x)=+\infty$ and $u_{*}(x)=-\infty$ throughout $\Omega$.

For the reader's convenience, we conclude with a summary of the main steps in our (at least theoretical) reconstruction of the nonlinear term $a(x, u)$ on $E$, assuming, for simplicity that $\partial \Omega$ is $C^{1,1}$ :

(i) Use formula (4.1) to determine $\Lambda_{q(\cdot ; \theta)}$ on $\partial \Omega$ for every $\theta \in \mathbb{R}$.

(ii) Let $\Omega$ be a $C^{1,1}$ domain containing $\partial \Omega$. Construct $\psi_{0}(x ; \theta)$ in $\Omega \backslash \Omega$ as in Lemma 3.2 .

(iii) With $q(\cdot ; \theta)$ defined to equal $\Delta \psi_{0}(\cdot ; \theta) / \psi_{0}(\cdot ; \theta)$ in $\Omega \backslash \Omega$, determine $\tilde{\Lambda}_{q(\cdot ; \theta)}$ on $\partial \tilde{\Omega}$.

(iv) Solve the integral equations (3.10) to obtain $\psi(x, k ; \theta)$ for $x$ on $\partial \tilde{\Omega}$ and then $t_{q(\cdot ; \theta)}(k)$ on $\mathbb{C} \backslash 0$ by formula $(3.11)$.

(v) Use the procedure given in [N 3] to recover $\psi_{0}(x ; \theta)$ for $x$ in $\Omega$ from $t$. Note that:

$$
\psi_{0}(x ; \theta) \equiv \frac{\partial u}{\partial \theta}(x ; \theta) \quad \text { for } x \in \Omega .
$$

(vi) Determine the function $\theta(x ; u)$ (inverse of $\theta \rightarrow u(x ; \theta)$ ), for instance by solving the ODE:

$$
\frac{\partial \theta}{\partial u}(x ; u)=\frac{1}{\psi_{0}(x ; \theta(x ; u))}, \quad \theta(x ; 0)=0,
$$

which blows up precisely at $u=u^{*}(x)$ and $u=u_{*}(x)$. Alternatively, one can integrate equation (4.22) in $\theta$ to find $u_{*}(x), u^{*}(x)$ and $u(x, \theta)$, then invert the latter.

(vii) Obtain the desired function $a(x, u)$ on $E$ from the formula (compare with (4.19)):

$$
a(x ; u)=\int_{0}^{\theta(x ; u)} \Delta \psi_{0}(x ; \theta) d \theta
$$




\section{APPENDIX}

We give here the scattering theory results needed to obtain Corollaries 1.4 and 1.5 from Theorem 1.3. The arguments are modifications to the case of nonconstant background of those in [N 1] and [N 2], and are the same for any dimension $n \geq 2$.

Let $\Omega$ be a bounded Lipschitz domain with connected exterior $\Omega^{e}=\mathbb{R}^{n} \backslash \bar{\Omega}$ and let $q$ be a real-valued potential satisfying the short-range condition (1.12). For every $f$ in $H^{1 / 2}(\partial \Omega)$ there is a unique outgoing solution $u^{e}(x ; \lambda ; f)$ to the exterior Dirichlet problem

$$
(-\Delta+q-\lambda) u^{e}=0 \text { in } \Omega^{e}
$$

with $\left.u^{e}\right|_{\partial \Omega}=f$. (Uniqueness follows from a classical result of Kato and existence can then be obtained by adapting an old argument of R. Phillips. We omit the details.) We define an exterior Dirichlet-to-Neumann map by

$$
\Lambda_{q-\lambda}^{e} f=\left.\frac{\partial u^{e}}{\partial \nu}(\cdot ; \lambda ; f)\right|_{\partial \Omega} .
$$

The main properties of the single-layer operator $\mathscr{S}_{q}(\lambda)$ needed here are given in the following proposition, which also makes clear the usefulness of $\Lambda_{q-\lambda}^{e}$.

Proposition A.1. Let $\Omega$ be a bounded Lipschitz domain with $\Omega^{e}=\mathbb{R}^{n} \backslash \bar{\Omega}$ connected, and let $q$ be a real-valued potential satisfying (1.12). Then $\mathscr{S}_{q}(\lambda)$ is a bounded operator: $H^{-1 / 2}(\partial \Omega) \rightarrow H^{1 / 2}(\partial \Omega)$ which is invertible if and only if $\lambda$ is not a Dirichlet eigenvalue of $-\Delta+q$ in $\Omega$, in which case we have

$$
\mathscr{S}_{q}^{-1}(\lambda)=\Lambda_{q-\lambda}-\Lambda_{q-\lambda}^{e} .
$$

Proof. We denote by $H_{\delta}^{s}\left(\mathbb{R}^{n}\right)$ the weighted Sobolev space with norm

$$
\|f\|_{H_{\delta}^{s}}=\left\|\langle x\rangle^{\delta} f\right\|_{H^{s}},\langle x\rangle=\left(1+|x|^{2}\right)^{1 / 2} .
$$

1. The Green's function $\mathscr{G}_{q}(x, y ; \lambda)$ is the kernel of the (boundary value of the) resolvent

$$
R_{q}(\lambda)=\lim _{\varepsilon \downarrow 0}[(-\Delta+q)-(\lambda+i \varepsilon)]^{-1}
$$

which is known to exist as a bounded operator: $L_{\delta}^{2}\left(\mathbb{R}^{n}\right) \rightarrow H_{-\delta}^{2}\left(\mathbb{R}^{n}\right)$ for every $\lambda>0$ and $\delta>\frac{1}{2}$ (combine the limiting absorption principle with absence of positive energy eigenvalues for the potentials considered here). By duality and interpolation we also have $R_{q}(\lambda)$ bounded: $H_{\delta}^{s}\left(\mathbb{R}^{n}\right) \rightarrow H_{-\delta}^{s+2}\left(\mathbb{R}^{n}\right)$ for all $s$ in $[-2,0]$.

For any $f$ in $H^{-1 / 2}(\partial \Omega)$ denote by $f d \sigma$ the single-layer distribution on $\mathbb{R}^{n}$ defined by

$$
\langle v, f d \sigma\rangle=\int_{\partial \Omega} v f d \sigma, \quad v \in H^{1}\left(\mathbb{R}^{n}\right) ;
$$

since $f d \sigma$ is in $H^{-1}\left(\mathbb{R}^{n}\right)$ and has compact support we can define the function

$$
\mathscr{S}_{q}(\lambda) f=R_{q}(\lambda)(f d \sigma) \in H_{-\delta}^{1}\left(\mathbb{R}^{n}\right) .
$$

Combining the above with the trace theorem shows that $\mathscr{S}_{q}(\lambda)$ is a bounded operator from $H^{-1 / 2}(\partial \Omega)$ to $H^{1 / 2}(\partial \Omega)$. 
2. Using the resolvent equation we have (as functions on $\mathbb{R}^{n}$ )

$$
\mathscr{S}_{q}(\lambda) f-\mathscr{S}_{0}(\lambda) f=R_{0}(\lambda) q \mathscr{S}_{q}(\lambda) f
$$

since the term on the right is in $H_{-\delta}^{2}\left(\mathbb{R}^{n}\right)$ it follows that the jump in the normal derivative of $\mathscr{S}_{q}(\lambda) f$ across $\partial \Omega$ is the same as that of $\mathscr{S}_{0}(\lambda) f$.

If $u$ is an interior Dirichlet eigenfunction then $\mathscr{S}_{q}(\lambda)\left(\left.\frac{\partial u}{\partial \nu}\right|_{\partial \Omega}\right)=0$ on $\partial \Omega$ so $\mathscr{S}_{q}(\lambda)$ is not injective in that case. Conversely, if $\mathscr{S}_{q}(\lambda) h=0$ on $\partial \Omega$ for some $h \not \equiv 0$, then the function $R_{q}(\lambda)(h d \sigma)$ is an interior eigenfunction with normal derivative $h$ (use the uniqueness of the exterior Dirichlet problem and the jump relation across $\partial \Omega$ ). So if $\lambda$ is not an interior eigenvalue, $\mathscr{S}_{q}(\lambda)$ is injective. To prove surjectivity in this case, as well as (A.3), we define, for $f$ in $H^{-1 / 2}(\partial \Omega)$ the double-layer distribution $d^{f}$ in $H^{-2}\left(\mathbb{R}^{n}\right)$ by

$$
\left\langle v, d^{f}\right\rangle=\int_{\partial \Omega} \frac{\partial v}{\partial \nu} f d \sigma, \quad v \in H^{2}\left(\mathbb{R}^{n}\right),
$$

and the double-layer potential

$$
\mathscr{D}_{q}(\lambda) f=R_{q}(\lambda)\left(d^{f}\right) \in L_{-\delta}^{2}\left(\mathbb{R}^{n}\right) .
$$

From the definitions we then obtain, for any $f$ in $H^{1 / 2}(\partial \Omega)$

and, similarly,

$$
\mathscr{S}_{q}(\lambda)\left(\Lambda_{q-\lambda} f\right)=\mathscr{D}_{q}(\lambda) f \text { in } \Omega^{e}
$$

$$
\mathscr{S}_{q}(\lambda)\left(\Lambda_{q-\lambda}^{e} f\right)=\mathscr{D}_{q}(\lambda) f \text { in } \Omega .
$$

Thus, for $f$ in $H^{1 / 2}(\partial \Omega)$, the function $\mathscr{D}_{q}(\lambda) f$ is piecewise $H^{1}$ and its jump across $\partial \Omega$ is (again using the resolvent equation) the same as that of $\mathscr{D}_{0}(\lambda) f$ :

$$
\mathscr{D}_{q}^{e}(\lambda) f-\mathscr{D}_{q}^{i}(\lambda) f=f,
$$

with $\mathscr{D}_{q}^{e} f\left(\mathscr{D}_{q}^{i} f\right)$ denoting the trace of $\mathscr{D}_{q}(\lambda) f$ on $\partial \Omega$ from $\Omega^{e}$ (respectively $\Omega$ ). Combining the identities (A.11), (A.12) with (A.13) yields

$$
\mathscr{S}_{q}(\lambda)\left(\Lambda_{q-\lambda}-\Lambda_{q-\lambda}^{e}\right) f=f,
$$

for any $f$ in $H^{1 / 2}(\partial \Omega)$. Thus $\mathscr{S}_{q}(\lambda)$ is surjective and (A.3) is established.

The proof of Corollary 1.4 is now immediate: assume $\mathscr{S}_{q_{1}}(\lambda)=\mathscr{S}_{q_{2}}(\lambda)$; since $\lambda<\lambda_{1}\left(q_{1}\right), \mathscr{S}_{q_{1}}(\lambda)$ is invertible, hence so is $\mathscr{S}_{q_{2}}(\lambda)$. Thus $\lambda$ is not a Dirichlet eigenvalue of $-\Delta+q_{2}$ in $\Omega$, and (A.3) holds for $q_{2}$ as well as for $q_{1}$. Since $q_{1}$ and $q_{2}$ agree outside $\Omega$, we have $\Lambda_{q_{1}-\lambda}^{e}=\Lambda_{q_{2}-\lambda}^{e}$, and the identity (1.15) follows. Theorem 1.3 now yields $q_{1}=q_{2}$.

To obtain Corollary 1.5 we define, as in [N2], the near-to-far-field operator $\mathscr{F}(\lambda): H^{1 / 2}(\partial \Omega) \rightarrow L^{2}\left(S^{n-1}\right)$ by

$$
\mathscr{F}(\lambda) f(\omega)=4 \pi(2 \pi i / \sqrt{\lambda})^{\frac{n-3}{2}} u_{\infty}(\omega ; \lambda ; f)
$$


with $u_{\infty}(\cdot ; \lambda ; f)$ the far-field pattern of the outgoing solution $u^{e}(\cdot ; \lambda ; f)$. (The normalization will become clear in Lemma A.3 below). Let $A_{q}(\lambda)$ denote the operator on $L^{2}\left(S^{n-1}\right)$ with kernel the scattering amplitude of $q$, and let $\mathscr{F}(\lambda)$ be the analogue of $\mathscr{F}(\lambda)$ corresponding to the incoming exterior solution.

Proposition A.2. Let $\Omega$ be a bounded Lipschitz domain with $\Omega^{e}=\mathbb{R}^{n} \backslash \bar{\Omega}$ connected. Let $q_{1}, q_{2}$ be two real-valued potentials which satisfy (1.12) and are identical outside $\Omega$. Then

$$
A_{q_{1}}(\lambda)-A_{q_{2}}(\lambda)=\mathscr{F}(\lambda)\left(\mathscr{S}_{q_{2}}(\lambda)-\mathscr{S}_{q_{1}}(\lambda)\right) \mathscr{F}_{-}^{*}(\lambda) .
$$

From Kato's theorem and unique continuation we know that $\mathscr{F}(\lambda), \mathscr{F}_{-}(\lambda)$ are injective, hence also that the range of $\mathscr{F}_{-}^{*}(\lambda): L^{2}\left(S^{n-1}\right) \rightarrow H^{-1 / 2}(\partial \Omega)$ is dense. Thus, if $A_{q_{1}}(\lambda)=A_{q_{2}}(\lambda)$, the identity (A.16) shows that $\mathscr{S}_{q_{1}}(\lambda)=$ $\mathscr{S}_{q_{2}}(\lambda)$ on $\partial \Omega$ and allows us to derive Corollary 1.5 from Corollary 1.4. For reconstruction purposes, the related identity (A.28) below may be preferred.

The proof of Proposition A.2 and of (A.28) will follow from the next two Lemmas, the first of which establishes an explicit integral formula for $\mathscr{F}(\lambda)$ in terms of the scattering solution $\varphi^{e}(x, \omega ; \lambda)$ of the exterior problem (A.1) with $\left.\varphi^{e}\right|_{\partial \Omega}=0$ and $\varphi^{e}(x, \omega ; \lambda)-\exp (i \sqrt{\lambda} x \cdot \omega)$ outgoing. (To allow for the limited decay assumption (1.12) on $q$, one thinks of $\varphi^{e}(x, \omega)$ as the kernel of an operator defined on $L^{2}\left(S^{n-1}\right)$ - see also (A.24) below.)

Lemma A.3. Let $\Omega$ be a bounded Lipschitz domain in $\mathbb{R}^{n}$ with connected exterior $\Omega^{e}$ and let $q$ be a real-valued potential on $\Omega^{e}$ satisfying (1.12). Then

$$
\mathscr{F}(\lambda) f(\omega)=\int_{\partial \Omega} \frac{\partial \varphi^{e}}{\partial \nu}(x,-\omega ; \lambda) f(x) d \sigma(x)
$$

(as functions in $L^{2}\left(S^{n-1}\right)$ ) for any $f$ in $H^{1 / 2}(\partial \Omega)$, while for any $g$ in $L^{2}\left(S^{n-1}\right)$

$$
\mathscr{F}_{-}^{*}(\lambda) g(x)=\int_{S^{n-1}} \frac{\partial \varphi^{e}}{\partial \nu}(x, \omega ; \lambda) g(\omega) d \sigma(\omega) .
$$

Proof. Let $\rho_{0}$ be such that $\bar{\Omega} \subset\left\{x:|x|<\rho_{0}\right\}$. Choose $\chi$ in $C^{\infty}\left(\mathbb{R}^{n}\right)$ vanishing in a neighborhood of $\bar{\Omega}$ and identically one for $|x|>\rho_{0}$. Then the function $\tilde{u}=\chi u^{e}$ satisfies $(\Delta+\lambda) \tilde{u}=v$, with

$$
v=(\Delta \chi+q \chi) u^{e}+2 \nabla \chi \cdot \nabla u^{e},
$$

and is outgoing, hence $\tilde{u}=-R_{0}(\lambda) v$. From the asymptotic behaviour of the latter we find

$$
\mathscr{F}(\lambda) f(\omega)=-\hat{v}(\sqrt{\lambda} \omega)=-\lim _{R \rightarrow \infty} \int_{|x|<R} e^{-i \sqrt{\lambda} \omega \cdot x}(\Delta+\lambda) \tilde{u}(x) d x,
$$

with the limit taken in the $L^{2}\left(S^{n-1}\right)$ norm. Integration by parts gives (A.21)

$$
\begin{aligned}
\mathscr{F}(\lambda) & f(\omega) \\
\quad & =-\lim _{R \rightarrow \infty} \int_{|x|=R}\left[e^{-i \sqrt{\lambda} \omega \cdot x} \frac{\partial u^{e}}{\partial \nu}(x ; \lambda ; f)-u^{e}(x ; \lambda ; f) \frac{\partial}{\partial \nu}\left(e^{-i \sqrt{\lambda} \omega \cdot x}\right)\right] d \sigma(x) .
\end{aligned}
$$


Since $\varphi^{e}(x,-\omega ; \lambda)-\exp (-i \sqrt{\lambda} \omega \cdot x)$ and $u^{e}(x ; \lambda)$ are both outgoing, the limit (A.21) equals

(A.22)

$$
\begin{aligned}
\mathscr{F}(\lambda) & f(\omega) \\
= & -\lim _{R \rightarrow \infty} \int_{|x|=R}\left[\varphi^{e}(x,-\omega ; \lambda) \frac{\partial u^{e}}{\partial \nu}(x)-u^{e}(x) \frac{\partial}{\partial \nu} \varphi^{e}(x,-\omega ; \lambda)\right] d \sigma(x),
\end{aligned}
$$

and (A.17) follows by another integration by parts.

To verify (A.18), let $\varphi_{-}^{e}(x, \omega ; \lambda)$ denote the exterior Dirichlet solution with $\varphi_{-}^{e}(x, \omega ; \lambda)-\exp (i \sqrt{\lambda} x \cdot \omega)$ incoming. Then $\overline{\varphi_{-}^{e}(x,-\omega ; \lambda)}-\exp (i \sqrt{\lambda} x \cdot \omega)$ is outgoing and uniqueness for the exterior problem shows (since $q$ is real-valued) that $\overline{\varphi_{-}^{e}(x,-\omega, \lambda)}$ and $\varphi^{e}(x, \omega, \lambda)$ are identical.

The standard scattering solution $\varphi_{q}^{+}(x, \omega ; \lambda)$ in $\mathbb{R}^{n}$ of the LippmannSchwinger equation

$$
\varphi_{q}^{+}(x, \omega ; \lambda)=\exp (i \sqrt{\lambda} x \cdot \omega)-R_{0}(\lambda)\left(q(\cdot) \varphi_{q}^{+}(\cdot, \omega ; \lambda)\right),
$$

can be constructed as the kernel of the map defined on functions in $L^{2}\left(S^{n-1}\right)$ by

$$
\int_{S^{n-1}} \varphi_{q}^{+}(x, \omega ; \lambda) g(\omega) d \sigma(\omega)=:\left(I-R_{q}(\lambda) q\right)\left(\int_{S^{n-1}} e^{i \sqrt{\lambda}(\cdot, \omega)} g(\omega) d \sigma(\omega)\right) .
$$

Lemma A.4. Under the hypotheses of Lemma A.3 we have the identity:

$$
\mathscr{S}_{q}(\lambda) \frac{\partial \varphi^{e}}{\partial \nu}(\cdot, \cdot ; \lambda)=\varphi_{q}^{+}(\cdot, \cdot ; \lambda)
$$

as operators: $L^{2}\left(S^{n-1}\right) \rightarrow H^{1 / 2}(\partial \Omega)$.

Proof. For any $g \in L^{2}\left(S^{n-1}\right)$, the function

$$
\int_{S^{n-1}}\left[\varphi_{q}^{+}(x, \omega ; \lambda)-\varphi^{e}(x, \omega ; \lambda)\right] g(\omega) d \sigma(\omega)
$$

is an outgoing solution of the exterior problem (A1) with trace

$$
\int_{S^{n-1}} \varphi_{q}^{+}(x, \omega ; \lambda) g(\omega) d \sigma(\omega)
$$

on $\partial \Omega$. Thus, from the definition of the exterior Dirichlet-to-Neumann map we have

(A.26) $\Lambda_{q-\lambda}^{e}\left(\varphi_{q}^{+}(\cdot, \omega ; \lambda)\right)=\frac{\partial}{\partial \nu}\left(\varphi_{q}^{+}-\varphi^{e}\right)=\Lambda_{q-\lambda}\left(\varphi_{q}^{+}(\cdot, \omega ; \lambda)\right)-\frac{\partial \varphi^{e}}{\partial \nu}(\cdot, \omega ; \lambda)$.

Applying $\mathscr{S}_{q}(\lambda)$ to both sides of (A.26) and using (A.3) yields (A.25).

Proof of Proposition A.2. Since the far-field pattern of

$$
\varphi_{q}^{+}(x, \omega ; \lambda)-\exp (i \sqrt{\lambda} x \cdot \omega)
$$

is given by the scattering amplitude, for every $g$ in $L^{2}\left(S^{n-1}\right)$ we have (using (A.23))

$(\mathrm{A} .27) \mathscr{F}(\lambda) \int_{S^{n-1}}\left[\varphi_{q_{1}}^{+}(\cdot, \omega ; \lambda)-\varphi_{q_{2}}^{+}(\cdot, \omega ; \lambda)\right] g(\omega) d \sigma(\omega)=\left[A_{q_{2}}(\lambda)-A_{q_{1}}(\lambda)\right] g$. 
Applying $\mathscr{F}(\lambda)$ to both sides of (A.25), using (A.18) and (A.27), yields (A.16).

If $A_{\Omega, q}(\lambda)$ denotes the scattering amplitude corresponding to the exterior Dirichlet problem, (that is, the operator on $L^{2}\left(S^{n-1}\right)$ with kernel the far-field pattern of $\left.\varphi^{e}(x, \omega ; \lambda)-\exp (i \sqrt{\lambda} x \cdot \omega)\right)$ then the above proof gives the identity

$$
\mathscr{F}(\lambda) \mathscr{S}_{q}(\lambda) \mathscr{F}_{-}^{*}(\lambda)=A_{\Omega, q}(\lambda)-A_{q}(\lambda),
$$

which can, in principle, be used to recover $q$ in $\Omega$ from knowledge of $A_{q}(\lambda)$ and of $q$ outside $\Omega$ (see also [N 2]).

\section{REFERENCES}

[A-S] M. Aizenman and B. Simon, Brownian motion and Harnack's inequality for Schrödinger operators, Comm. Pure Appl. Math. 35 (1982), 209-273.

[B] Yu. M. Berezanskii, Trudy Moskov. Mat. Obshch. 7 (1958), 3-62.

[G-T] D. Gilbarg and N. Trudinger, Elliptic partial differential equations of second order, Springer-Verlag, 1977.

[Is 1] V. Isakov, Completeness of products of solutions and some inverse problems for PDE, J. Differential Equations 92 (1991), 305-317.

[Is 2] _ On uniqueness in inverse problems for semilinear parabolic equations, Arch. Rational Mech. Anal. 124 (1993), 1-13.

[Is-Su] V. Isakov and Z. Sun, The inverse scattering at fixed energies in two dimensions, Indiana Univ. Math. J. (1995) (to appear).

[Is-Sy] V. Isakov and J. Sylvester, Global uniqueness for a semilinear elliptic inverse problem, Comm. Pure Appl. Math 47 (1994), 1403-1410.

[N 1] A.I. Nachman, Reconstructions from boundary measurements, Ann. of Math. 128 (1988), 531-577.

[N 2] _ Inverse scattering at fixed energy, Proc. 10th International Congress on Math. Phys., Leipzig 1991, edited by K. Schmüdgen, Springer-Verlag, 1992, pp. 434-441.

[N 3] Global uniqueness for a two-dimensional inverse boundary value problem, Univ. of Rochester, Dept. of Math. Preprint Series 19, 1993;Ann. of Math. (1995) (to appear).

[No] R. G. Novikov, The inverse scattering problem on a fixed energy level for the two dimensional Schrödinger operator, J. Funct. Anal 103 (1992), 409-463.

[S 1] Z. Sun, On an inverse boundary value problem in two dimensions, Comm. Partial Differential Equations 14 (1989), 1101-1113.

[S 2] - On a quasilinear inverse boundary value problem, Math. Z. (1995) (to appear).

[Su-U1] Z. Sun and G. Uhlmann, Generic uniqueness for an inverse boundary value problem, Duke Math. J. 62 (1991), 131-155.

[Su-U2] _ Recovery of singularities for formally determined inverse problems, Comm. Math. Phys. 153 (1993), 431-445.

[Sy-U] J. Sylvester and G. Uhlmann, $A$ uniqueness theorem for an inverse boundary value problem in electrical prospection, Comm. Pure Appl. Math 39 (1986), 92-112.

Department of Mathematics and Statistics, Wichita State University, Wichita, KANSAS 67260-0033

E-mail address: isakov@twsum.uc.twsu.edu

Department of Mathematics, University of Rochester, Rochester, New York 14627

E-mail address: adnm@clara.math.rochester.edu 